\title{
Effect of smoking cessation in saliva compounds by FTIR spectroscopy
}

\author{
Laís Morandini Rodrigues ${ }^{\mathrm{a}, *}$, Taciana Deprá Magrini ${ }^{\mathrm{b}}$, Celina Faig Lima ${ }^{\mathrm{c}}$, Jaqueline Scholz ${ }^{\mathrm{d}}$, \\ Herculano da Silva Martinho ${ }^{\mathrm{e}}$, Janete Dias Almeida ${ }^{\mathrm{c}}$ \\ a Department of Biosciences and Oral Diagnosis, Institute of Science and Technology, UNESP - Univ Estadual Paulista, Brazil. Biologic E' Materials Sciences, Division of Prosthodontics, \\ University of Michigan, United States \\ ${ }^{\mathrm{b}}$ Instituto de Pesquisa e Desenvolvimento, Univ do Vale do Paraíba, Brazil

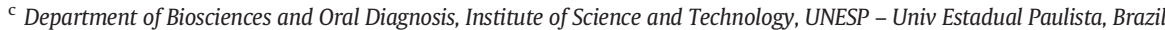 \\ d Smoking Cessation Program Department, Heart Institute, University of Sao Paulo Medical School, Brazil \\ e Centro de Ciências Naturais e Humanas, Universidade Federal do ABC, Brazil
}

\section{A R T I C L E I N F O}

\section{Article history:}

Received 20 July 2016

Received in revised form 15 October 2016

Accepted 9 November 2016

Available online 23 November 2016

\section{Keywords:}

Saliva

FTIR spectroscopy

Tobacco

Smoking cessation

\begin{abstract}
A B S T R A C T
Introduction: Smoking is currently considered one of the biggest risk factors for the development of various diseases and early death. Fourier transform infrared (FTIR) spectroscopy is a valuable tool for analysis of biofluids such as saliva and is considered useful for diagnostic purposes. The aim of this study was to evaluate the effect of smoking cessation on saliva composition by FTIR spectroscopy.

Methods: We analyzed the saliva of participants in two groups: a smoker group made up of 10 chronic smokers and a former smoker group made up of 10 individuals who had stopped smoking. Members of both groups had similar smoking history.

Results: The results showed few differences in spectral intensity between the groups; however, spectral peaks were slightly increased in the group of smokers in the bands for DNA, indicating modification of its content or cell necrosis. They were also increased for the mannose-6-phosphatase molecule, which is expressed in prostate and breast carcinomas. In the former smoker group, the peak of thyociante was decreased and the band referring to collagen increased in intensity, which indicates a better tissue regeneration capacity.

Conclusion: Considering these results and the fact that tobacco intake was similar between the groups, it can be concluded that there was recovery of tissue regeneration capacity with smoking cessation during the study period, although the effects found in smokers persisted in the bodies of those who had given up smoking.
\end{abstract}

(c) 2016 Elsevier B.V. All rights reserved.

\section{Introduction}

Saliva is a complex body fluid [1] that contains a large number of different types of proteins, hormones and ions [2]. It is secreted primarily by three pairs of salivary glands (parotid, submandibular and sublingual), as well as numerous minor salivary glands [2], With the continuous advancement of proteomics technology, salivary diagnosis has become a focus of several studies [2] and, mainly because besides being considered a fluid with excellent indicators of plasma levels of various substances [1] its collection is simple and noninvasive. Thus, saliva has been used for the early diagnosis, prevention, and monitoring process of various diseases $[2,3,4]$.

\footnotetext{
* Corresponding author at: Department of Biosciences and Oral Diagnosis, Institute of Science and Technology, UNESP - Univ Estadual Paulista, Av. Francisco José Longo, 777 São Dimas, 12245-000 São José dos Campos, São Paulo, Brazil.

E-mail addresses: lais.morandini@gmail.com (L.M. Rodrigues), janete@fosjc.unesp.br (J.D. Almeida).
}

In recent years, various spectroscopic techniques have been studied in the biodiagnosis area and have greatly advanced in medical and diagnostic ability [5]. Fourier transform infrared (FTIR) spectroscopy is a vibrational spectroscopy method that can be used for qualitative and structural analysis and to determine structural changes in organic molecules [6]. It has been considered a powerful tool to analysis of biological samples such as plasma, serum, tissue, saliva, and urine and is already offered as a complementary technique for the clinical diagnosis and characterization of various types of diseases [6], including lesions which early diagnosis is a significant prognostic factor [7].

Habitual smoking is the most significant threat to the world's population, accounting for $30 \%$ of early death [8]. It is the biggest risk factor for cardiovascular disease and chronic obstructive pulmonary diseases $[9,10]$ and malignancies [11], mainly because the substances contained in tobacco affect and damage several organs and tissues [8].

Since it is known the damage caused by tobacco systemically, the aim of this study was to compare the saliva of patients who smoke and patients who discontinued tobacco use for a period of at least 6 months and up to 3 years through FTIR. We proposed demonstrate 
significant differences in salivary content, detecting any changes at the molecular level as results of tobacco cessation, and then prove that the period during which the individual did not use cigarettes was sufficient to change the saliva composition and consequently general healthy.

\section{Material and Methods}

There were two groups of participants from the Smoking Cessation Program, Heart Institute (InCor), University of Sao Paulo Medical School, São Paulo, Brazil. The study was approved by the Ethics Committee of the Institute of Science and Technology, UNESP - Univ Estadual Paulista, São Paulo, Brazil, Protocol CAEE 45703114.6.0000.0077. It was conducted in accordance to Helsinki Declaration. Informed consent was obtained from all subjects prior to their participation in the study. All participants were subjected to extra- and intra-oral clinical examination. Patients were considered eligible for enrolment into the research if they fulfilled the following inclusion criteria: no history of malignant neoplasms and maximum weekly intake of 3 alcoholic drinks. The exclusion criterion was the presence of any visible sign of intra-oral alteration. Each group was composed by the selection of 20 consecutive patients as follows:

- Smoker group: 10 chronic smokers with consumption equal to or more than 20 cigarettes/day for more than 10 years;

- Former smoker group: 10 subjects who had stopped smoking for a period of at least 6 months and a maximum of 3 years;

Participants also completed a questionnaire regarding tobacco smoking. Data was used to calculate the smoking history according to Faria et al. [12]: number of cigarettes smoked per day divided by 20 ( 1 pack has 20 cigarettes in Brazil) multiplied by the total number of years of smoking (results in packs/year). Exhaled carbon monoxide (CO) concentrations were measured using a mini Smokerlyzer [13] to verify the accuracy of information on the smoking cessation rate.

\subsection{Collection and Storage of Samples}

Patients were instructed to refrain from eating, drinking and brushing their teeth for at least $1 \mathrm{~h}$ prior to saliva collection.

The gathering was held in the afternoon between 1 p.m. and 3 p.m. hours. Unstimulated saliva was collected from participants by spitting [14] into a sterile collection container. After collection, the samples were immediately stored in a cryogenic Nalgene ${ }^{\circledR}$ tube in the freezer $\left(-80^{\circ} \mathrm{C}\right)$. The previously prepared samples were transported into dry ice container $\left(-20^{\circ} \mathrm{C}\right)$, to the spectroscopy laboratory of the Federal University of $A B C$ to be analyzed in an FTIR Spectrometer 660 - Varian Inc.

For analysis, samples were thawed at room temperature, and $30 \mu \mathrm{L}$ were placed in a saliva sample portand placed in an oven for drying at $40^{\circ} \mathrm{C}$ forming a thin film. This was necessary in order to reduce interference of water in the spectra acquisition.

\subsection{Obtaining and Analysis of FTIR Spectra}

The spectra were measured under the parameters of acquisition of 800 background scans, 4 min, background time, 200 scans, $4 \mathrm{~cm}^{-1}$ resolution, and $1 \mathrm{~min}$ total scan time (average). The measurement was performed by diffuse reflectance. It was obtained two to four spectra by sample (by participant), depending on the thickness and concentration of the film. From one of the samples of the former smoker group, it was not possible to obtain spectra. After correction and normalization and since spectra were very similar intra group (verified by PCA analysis, Supplementary Fig. 1) they were averaged by volunteer thus making possible a better understanding of the differences between the groups. Statistics was performed with 19 spectra (10 were from smoker group and 9 from the former smoker group).

Spectra were baseline corrected and vector normalized in Labspec 6 software. The regions of 400-750, 2270-2400 and 3700-4000 $\mathrm{cm}^{-1}$ were excluded from the principal component analysis (PCA). It was performed with the Minitab 17 software to originate a scatter plot and the loading plots from which it is inferred the main bands that are responsible for the separation between the samples. A binary logistic regression calculation was made from the Concordant Pairs PC1, PC2 and PC3, and it was obtained the concordant pairs percentage and the Pearson $P$-value. Thus, a receiver operating characteristic (ROC) curve graph was created, which gives the sensitivity and specificity of FTIR spectroscopy. For this calculation, it was used the PC1 and PC2 for reference, the state value used was the former smoker group, the threshold method was interpolation of data points, and the level of confidence was 95\%. The Origin Lab 8.5 software was used to calculate the area under the curve (a. u. c.) between the $2000-2115 \mathrm{~cm}^{-1}$ of the 19 spectra as well as the standard error for calculating the ROC curve as well as making the graphs. $t$-test of the areas was calculated two-tailed with unequal variance in Excel 2003.

\section{Results}

\subsection{Sample Characterization}

The smoker group consisted of 6 men and 5 women with a mean age of $54.55 \pm 10.85$ years $($ minimum $=33$ and maximum $=72$ ). The participants of this group smoked $25.64 \pm 7.94$ cigarettes/day for $35 \pm 13.95$ years, and tobacco intake was $43.68 \pm 17.87$ packs/ year. The average $\mathrm{CO}$ ratio was $9.72 \pm 2.8$.

The former smoker group consisted of 2 men and 8 women with a mean age of $55.50 \pm 11.1$ years $($ minimum $=31$ and maximum $=$ 67). Participants of this group smoked $26 \pm 13.10$ cigarettes/day for $31.20 \pm 13.86$ years, but reported cessation was of $12.5 \pm 9.31$ months. Tobacco intake for this group was $43.20 \pm 34$ packs/year. The average CO ratio was $2 \pm 0.66$.

\subsection{Analysis of the FTIR Spectra}

Fig. 1A and B shows the raw spectra from each group and Fig. 1C shows the average of each group. It was possible to verify that there is no great difference in intensity of the mean spectra between groups analyzed. However, we observed that in the bands between the range of $1028-1160 \mathrm{~cm}^{-1}$ (shoulder at $1037 \mathrm{~cm}^{-1}$ and peaks at 1075,1121 and $1170 \mathrm{~cm}^{-1}$ ) and $2000-2115 \mathrm{~cm}^{-1}$ (peak at $2058 \mathrm{~cm}^{-1}$ ), also in 1735 and $1750 \mathrm{~cm}^{-1}$ the intensity of spectra in the smoker group is slightly higher than the other group. However, from 1240 to $1670 \mathrm{~cm}^{-1}$ (peaks at 1314, 1341, 1401, 1455, 1542 and $1653 \mathrm{~cm}^{-1}$ ) the intensity of spectra in the former smoker group is slightly higher. With this information it was possible to build Table 1 with information regarding the vibrational assignments (Table 1). It can be inferred by the intensity of the bands or peaks, the greater amount of polysaccharides, nucleic acids, and thiocyanate in the smoker group saliva than in the former smoker group. In the former smoker group, proteins in general are in greater amount than in the smoker group.

Since the differences in the average spectra are slight, we recurred to PCA and binary logistic regression to identify with more precision the changes between the groups. The principal components analysis (PCA) and loadings allowed us to evaluate which wavenumbers and structural components were more relevant for the discrimination between samples analyzed. It was found that the principal components (PCs) PC1 contains $98.2 \%$ of the data, PC2 $1 \%$ and PC3 $0.2 \%$. In this study, PC1 and PC2 had 71\% of concordant pairs and 0.25 Pearson $P$ value as verified by binary logistic regression (Fig. 1A). The PC2 loading 


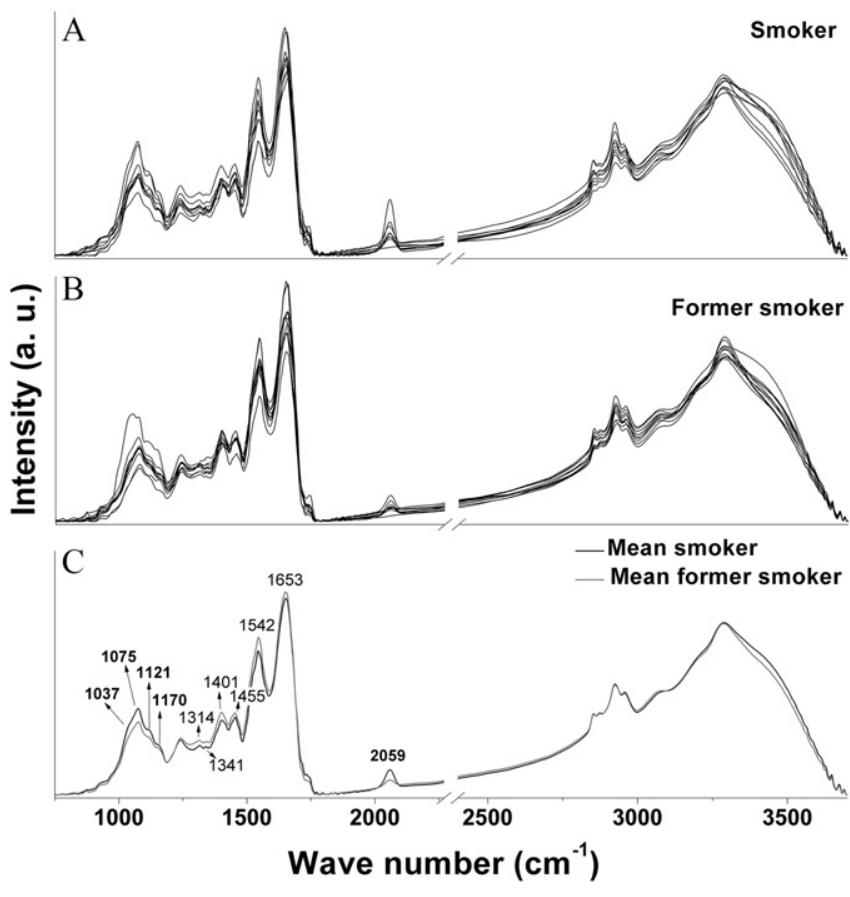

Fig. 1. Raw spectra of the smoker group (A) and the former smoker group (B) after baseline correction and vector normalization, and the mean spectra of both groups with the indication of the peaks which the intensity varies (C).

plot indicates that the main bands responsible for the positioning of the smoker group in the negative portion of the scatter plot is precisely the range from 1220 to $1700 \mathrm{~cm}^{-1}$, specifically the shoulders in 1392,1443 , $1511 \mathrm{~cm}^{-1}$ and the peaks in 1538,1544 and $1640 \mathrm{~cm}^{-1}$ (Fig. 2B). The region responsible for the positioning mostly of the former smoker group in the positive portion of PC2 scatter plot is from 980 to $1172 \mathrm{~cm}^{-1}$, specifically the peaks in 1078 and $1125 \mathrm{~cm}^{-1}$, the region from 2000 to $2115 \mathrm{~cm}^{-1}$, peak in $2058 \mathrm{~cm}^{-1}$. This means the ranges which have showed differences in the mean spectra of groups are almost the same responsible for discrimination in PC1 $\times$ PC2.

The PC2 and PC3 had 73\% of concordant pairs and 0.23 Pearson $P$-value (Fig. 2C). In this scatter plot the smoker group data are more spread than the former smoker, which is mostly clustered in the negative portion of PC2 because of the band in $1544 \mathrm{~cm}^{-1}$ and the shoulder in $1396 \mathrm{~cm}^{-1}$. The positive portion of PC3 loading shows the responsible bands for this arrangement are the shoulders in 1573 and in $1692 \mathrm{~cm}^{-1}$ (Fig. 2D). These bands mostly related to the amount of proteins in the former smoker group are important for the discrimination between both studied groups and highlight the difference in the chemical composition between them.

The band in $2058 \mathrm{~cm}^{-1}$ is due to thiocyanate. It has been shown in literature the a.u.c. is proportional to the amount of thiocyanate in saliva [15]. Since the band in $2058 \mathrm{~cm}^{-1}$ seems to be important for the discrimination between groups, we gave special attention to it by calculating the area under the curve (a. u. c.) in the range $2000-2115 \mathrm{~cm}^{-1}$, which is presented in the Fig. 3. It can be seen the average in the smoker group is greater than the former smoker group. In this case, $p$-value is equal to 0.05 . This result is expected since the level of thiocyanate in smokers was found greater than in non-smokers in the literature [16, 17]. It shows an indication of recovery of the former smokers after a period of time of 6 months to 3 years.

Fig. 3 presents the ROC curve for the method, calculated from PC1 and PC2, which gives a fair accuracy of the FTIR tool to discriminate between smoker and former smoker with an area under the curve of 0.72 (Fig. 4).

\section{Discussion}

Smoking is a global problem of civilization, and the estimated number of tobacco smokers is about 1.3 million $[18,19]$. Cigarette smoke contains more than 4800 chemicals, including 69 carcinogens [20], which seem to be of crucial importance in the development of various diseases [21] such as cardiovascular disease, accidental stroke, peripheral vascular disease, carcinoma of the lung, mouth, larynx, esophagus, stomach, kidney, bladder, and possibly others, as well as premature aging of the skin, osteoporosis, and impaired fertility, including impotence in men [11].

Before the damage that smoking can cause was known, it was proposed to analyze the saliva of smokers and former smokers to see if there are changes at the molecular level that could differentiate the quality of saliva and demonstrate how long the harmful effects of smoking may remain in the body in a short period of time. Saliva was chosen for this work because it can easily be collected using a noninvasive technique and several studies conducted with this biofluid have

Table 1

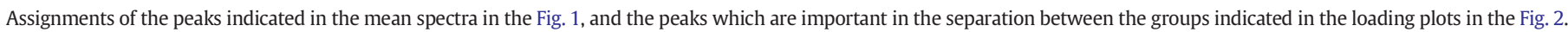

\begin{tabular}{|c|c|c|c|c|c|}
\hline \multicolumn{3}{|c|}{ Greater intensity in smoker mean spectra } & \multicolumn{3}{|c|}{ Greater intensity in former smoker mean spectra } \\
\hline Wavenumber $\left(\mathrm{cm}^{-1}\right)$ & Vibrational mode & Molecular group & Wavenumber $\left(\mathrm{cm}^{-1}\right)$ & Vibrational mode & Molecular group \\
\hline SH 1037 & $v \mathrm{CC}, v \mathrm{CH}_{2} \mathrm{OH}, v \mathrm{CO}$ & Glycogen; cellulose & 1314 & Amide III & Proteins; collagen \\
\hline 1075 & $\nu \mathrm{CN} ; v_{\mathrm{s}} \mathrm{PO}_{2}^{-}$ & RNA,DNA & 1341 & $\mathrm{CH}_{2}$ & collagen \\
\hline 1121 & $\begin{array}{l}\text { Symmetric phosphodiester bond } \\
\text { stretching }\end{array}$ & RNA & 1401 & $\delta_{\mathrm{s}} \mathrm{CH}_{3}$ & Methyl groups of proteins \\
\hline 1170 & $\nu \mathrm{CO}$ & - & 1455 & $\delta_{\mathrm{as}} \mathrm{CH}_{3}$ & “ \\
\hline \multirow[t]{2}{*}{2058} & $\mathrm{SCN}^{-}$ & Thiocyanate & 1542 & Amide II & Proteins \\
\hline & & & 1653 & Amide I & Proteins \\
\hline \multicolumn{3}{|c|}{ Positive region of PC2 loading } & \multicolumn{3}{|c|}{ Negative region of PC2 loading } \\
\hline 1078 & $v_{\mathrm{s}} \mathrm{PO}_{2}^{-}$ & RNA, DNA & SH 1392 & $\mathrm{CH}_{3}$ bending & Methyl groups of proteins \\
\hline 1125 & $v \mathrm{CC}, v \mathrm{CO}$ & Cellulose; sucrose & SH 1443 & $\delta \mathrm{CH}_{2}, \delta \mathrm{CH}$ & Cellulose; fatty acids; pectin \\
\hline \multirow[t]{4}{*}{2058} & $\mathrm{SCN}^{-}$ & Thiocyanate & SH 1511 & $\mathrm{CH}$ bending; $\nu \mathrm{C}=\mathrm{C}$; Amide II & $\begin{array}{l}\text { Phenyl rings; carotenoid; } \\
\text { proteins }\end{array}$ \\
\hline & & & 1538 & $\nu \mathrm{C}=\mathrm{N}, \nu \mathrm{C}=\mathrm{C} ;$ Amide II & Guanine; proteins \\
\hline & & & 1544 & Amide II & Proteins \\
\hline & & & 1640 & Amide I & Proteins \\
\hline \multicolumn{3}{|c|}{ Negative region of PC2 loading } & \multicolumn{3}{|c|}{ Positive region of PC3 loading } \\
\hline 1573 & $\mathrm{C}=\mathrm{N}$ & Adenine & SH 1396 & $\mathrm{CH}_{3}$ bending & Methyl groups of proteins \\
\hline 1692 & Antiparallel $\beta$-sheet of amide I & Proteins & 1544 & Amide II & Proteins \\
\hline
\end{tabular}

$\mathrm{SH}=$ shoulder, $v_{\mathrm{s}}=$ symmetric stretching, $v_{\mathrm{as}}=$ asymmetric stretching, $\delta_{\mathrm{s}}=$ symmetric wagging, $\delta_{\mathrm{as}}=$ asymmetric wagging. 

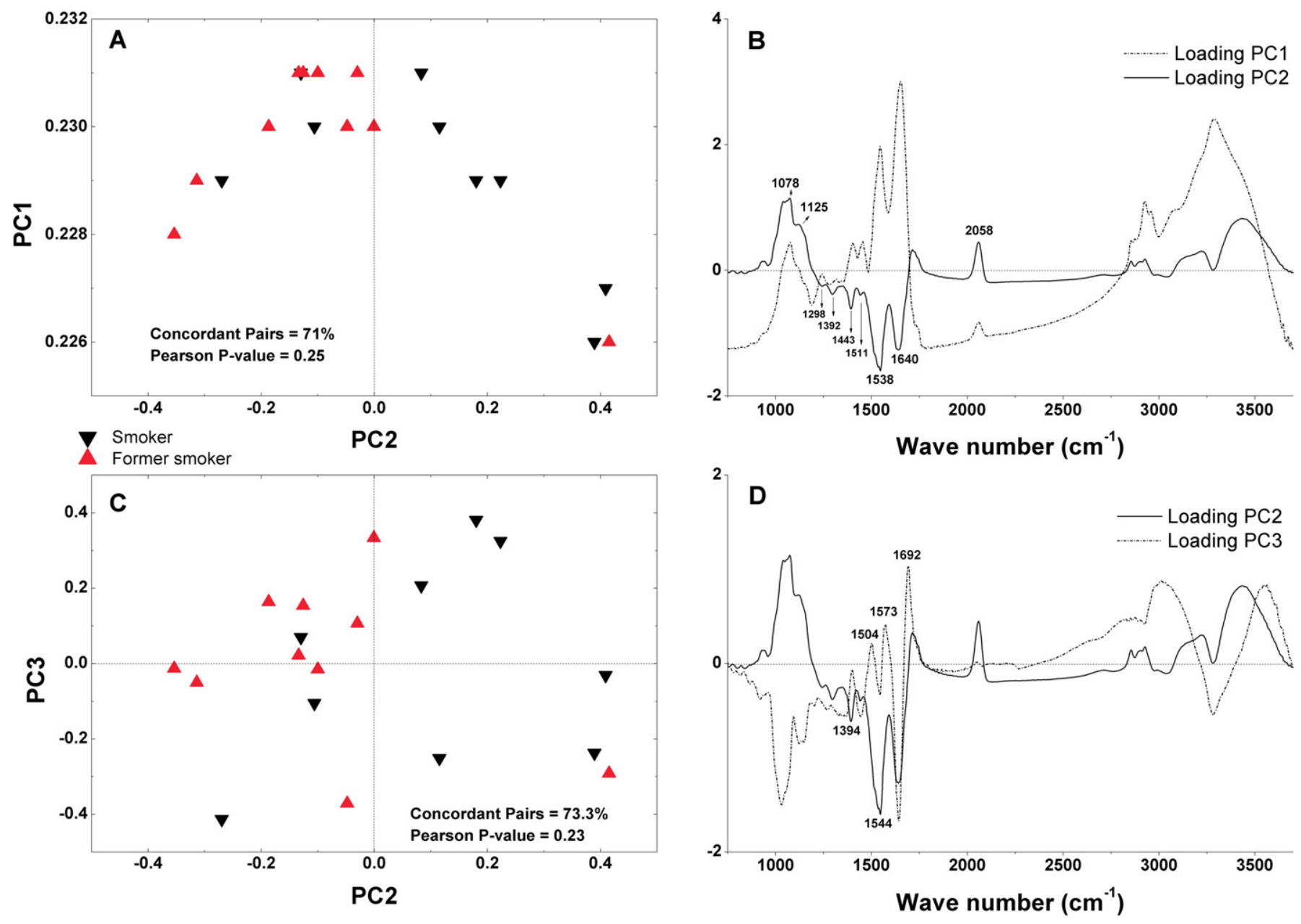

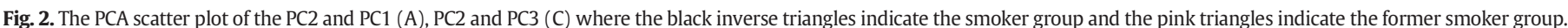

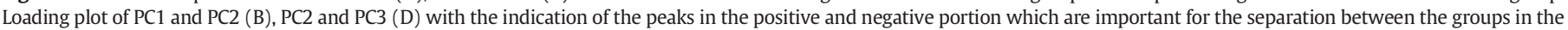
scatter plot.

shown efficacy for diagnosis $[3,22,4]$. In addition, studies have shown that the saliva content of the changes is closely related to the onset of oral diseases [2,23,24] and systemic diseases [2].

It is known that substances present in cigarettes, such as nicotine, can be detected in saliva [25], and this occurs because of the solubility properties of the agents present in cigarette smoke [26]. Moreover, it is clear that these substances interact with cells and tissues of the oral

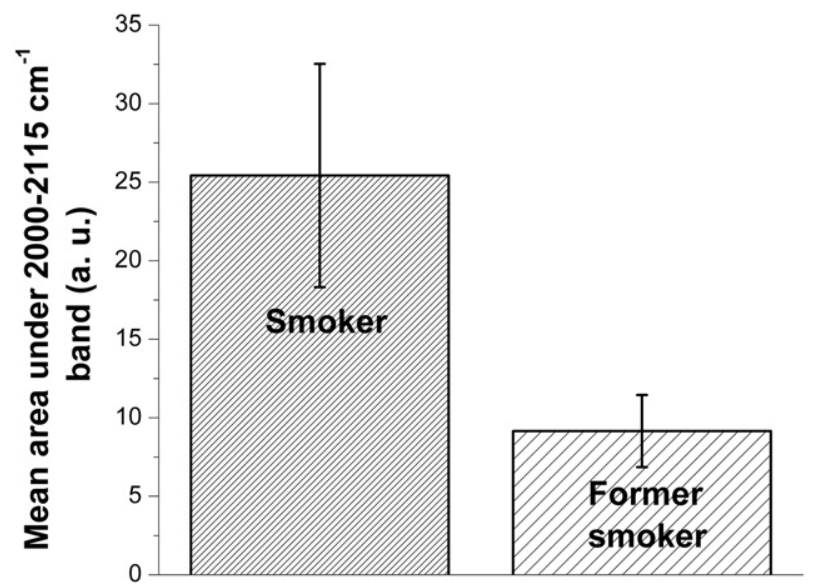

Fig. 3. Mean area under the band between 2000 and $2115 \mathrm{~cm}^{-1}$ of the smoker and former smoker group. cavity [26], can diffuse through biological membranes, and may even reach the deepest layers of tissue [27].

The spectra of saliva contains information about the molecular vibrations corresponding mainly to protein in the fingerprint region $\left(800-1800 \mathrm{~cm}^{-1}\right)$, and to the lipids in the higher wavenumbers (2500-3700). Analysis indicated that there was little difference in

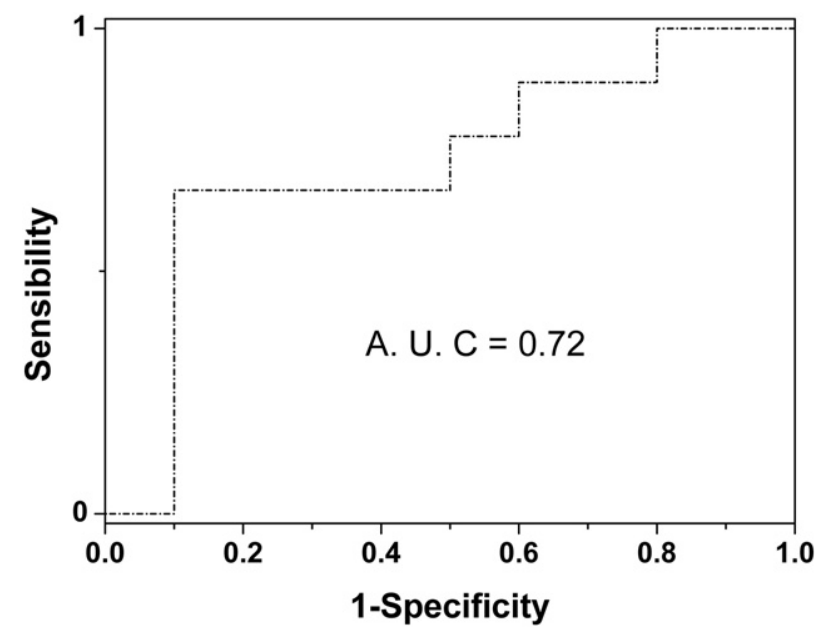

Fig. 4. Curve ROC made from $\mathrm{PC} 1$ and $\mathrm{PC} 2$. It shows a reasonable accuracy of the method with the a.u.c. $>0.7$. 
the average spectra intensity of fingerprint region and no difference in the high wavenumbers region; the intensity was the same, enabling us to conclude that the composition of saliva remained unchanged in the high wave numbers region, which was confirmed by the PCA analysis.

For the fingerprint region, it can be seen that the peaks in the region of $878 \mathrm{~cm}^{-1}-1176 \mathrm{~cm}^{-1}$ referred mainly to the presence of DNA and RNA, and its quantity was slightly higher for the group of smokers. This may be due to hypotheses such as necrosis and therefore desquamation of mucosal cells, since studies show that cigarette smoke induces cell necrosis [28], and therefore the presence of DNA in saliva could be present as the product of cell degradation.

Moreover, we cannot rule out, mainly by smoking contain carcinogenic substances, that the slightly larger spectral peaks of DNA could be linked to mutations and nuclear changes such as decreased apoptosis $[29,30]$, multinucleation and abnormal increase in mitotic activity, which are often observed and established in potentially pre malignant and malignant disorders such as leukoplakia, erythroplakia, oral submucous fibrosis $[31,32,33]$. Some studies have focused on the evaluation of the adduct cellular DNA content in smokers and nonsmokers $[34,35,36,37]$, and the results were unanimous that this molecule was increased in smokers. This corroborates the findings of this study; even though the spectral intensity difference is slightly higher in smokers, the DNA content is changed. Furthermore, it should be considered that both groups have much the same smoking history, which reflects the degree of tobacco exposure throughout the life of the individual, and this small difference found in the spectral peaks shows that even when cigarette use is discontinued, the negative effects do not remain in the body, significantly changing the final composition of saliva.

For former smokers, there were peaks of slightly larger intensity at $1314 \mathrm{~cm}^{-1}$ and $1341 \mathrm{~cm}^{-1}$, which refer to collagen. In the oral cavity, the collagen-producing fibroblast cell is physiologically responsible for wound repair [27]. The healing process is divided into steps, and the proliferative phase consists of four basic steps, epithelialization, angiogenesis, granulation tissue formation, and collagen deposition. Therefore, a small increase in collagen bands may mean that the cessation of cigarette use contributes to improving inflammation tissue regeneration capacity. Some studies have shown that nicotine and cigarette smoke condensate are capable of inhibiting the proliferation of fibroblasts and their ability to alter the production of proteolytic enzymes [38]. In their study, Silva et al. [27], found that the concentration of cigarette smoke condensate decreases cell viability of fibroblasts, inhibits cell migration, and interferes with the differentiation of myofibroblasts. These results help to clarify the differential role of cigarette smoke during the healing process. The spectral peaks at $1402,1455,1542$ and $1653 \mathrm{~cm}^{-1}$ refer to proteins in general which are present in the final composition of saliva, and were decreased in smoker group. Differences in these bands were found between former smokers for Ozek et al., [39], when they evaluated saliva of patients with chronical and aggressive periodontitis by FTIR spectroscopy. Similarly to the present study, they found a decrease of proteins in smoker patients and assigned increased oxidation, which is associated with smoking-enhanced reactive oxygen species in saliva. The spectral peak at $2058 \mathrm{~cm}^{-1}$ corresponds to the presence of thiocyanate (SCN-) previously described by Schultz et al. [15]. Our review shows greater discrepancy in this band between former smoker and smoker group. According to Weuffen et al. [40], low levels of $\mathrm{SCN}$ - are normally present in human body fluids (e.g. serum, saliva, urine) produced during digestion of some vegetables or by intake of thiocyanate-containing foods such as milk and cheese, however, the concentration level of thiocyanate in biological fluids increases significantly when there is exposure to hydrogen cyanide found in cigarettes [41].

We do not know exactly the role of the inorganic component of saliva, but some studies have reported that $\mathrm{SCN}^{-}$can cause neurological, endocrine alterations in the body and also be a factor in delayed wound healing $[42,43]$. Once established that $\mathrm{SCN}^{-}$has a negative effect on the inflammation process, we can also conclude, based on data found in this study in relation to the collagen bands, that the smoker group has poor healing process in relation to the former smoker group, and that smoking cessation aids in the recovery of cellular activities in the inflammatory process.

\section{Conclusion}

FTIR spectroscopy allowed us to evaluate the quality of the saliva of smokers and former smokers and identify significant differences in the final composition between groups and important information regarding the saliva quality in minimum time set. It was important to understand that even in a short period of time, the saliva of former smokers proved to be qualitatively better, mainly in collagen bands. This allows concluding that even in a short period of time, smoking cessation brings benefits to oral health. The technique was effective for characterizing the samples and could be used as a tool for analysis of saliva and monitoring of smokers who are more vulnerable to the development of pathologies that can arise from the smoking habit.

Supplementary data to this article can be found online at doi:10. 1016/j.saa.2016.11.009.

\section{Competing interests}

The authors state that they have no potential conflict of interest.

\section{Funding}

This work was supported by São Paulo Research Foundation (FAPESP) [grant number 13/02168].

\section{References}

[1] C. Llena-Puy, The role of saliva in maintaining oral health and as an aid to diagnosis, Med. Oral Patol. Oral Cir. Bucal. 11 (449) (2006) 455.

[2] Y. Sun, W. Du, C. Zhou, Y. Zhou, Z. Cao, Y. Tian, Y. Wang, Computational method for predict of saliva-scretory proteins and its apllications to identication of head and neck cancer biomarkers of salivary diagnosis, IEEE Trans. Nanobioscience 14 (2015) 167-174.

[3] A. Zhang, H. Sun, X. Wang, Saliva metabolomics opens door to biomarker discovery, disease diagnosis, and treatment, Appl. Biochem. Biotechnol. 168 (2012) 1718-1727.

[4] Y.H. Lee, D.T. Wong, Saliva: an emerging biofluid for early detection of diseases, Am. J. Dent. 22 (2009) 241-248.

[5] F. Zapata, M.A.F. de La Ossa, C. García-Ruiz, Emerging spectrometric techniques for the forensic analysis of body fluids, TrAC Trends Anal. Chem. 64 (2015) 53-63.

[6] O.G. Ildiz, M. Arslan, O. Unsalan, et al., FT-IR spectroscopy and multivariate analysis as an auxiliary tool for diagnosis of mental disorders: bipolar and schizophrenia cases, Spectrochim. Acta A Mol. Biomol. Spectrosc. 152 (2015) 551-556.

[7] Z. Udi, B. Eyal, B. Omri, et al., Early detection of breast cancer using total biochemical analysis of peripheral blood components: a preliminary study, BMC Cancer 15 (2015) 408.

[8] M. Demirtaș, Ü. Senel, S. Yüksel, M. Yüksel, A comparison of the generation of free radicals in saliva of active and passive smokers, Turk. J. Med. Sci. 44 (2014) 208-211.

[9] F. Khand, S.S. Shaikh, M.A. Ata, S.S. Shaikh, Evaluation of the effect of smoking on complete blood counts, serum C-reactive protein and magnesium levels in healthy adult male smokers, J. Pak. Med. Assoc. 65 (2015) 59-61.

[10] S.K. Das, Harmful health effects of cigarette smoking, Mol. Cell. Biochem. 253 (2003) 159-165.

[11] A.W. Musk, N.H. de Klerk, History of tobacco and health, Respirology 8 (2003) $286-290$.

[12] C.S. Faria, C. Botelho, R.M.V.G. da Silva, M.G. Ferreira, Smoking and abdominal fat in blood donors, J. Bras. Pneumol. 38 (2012) 356-363.

[13] J.S. Issa, N. Forti, S.D. Giannini, J. Diament, Intervenção sobre Tabagismo Realizada por Cardiologista em rotina Ambulatorial, Arq. Bras. Cardiol. 70 (1998) 271-274.

[14] P.P.A. Santos, D.P.P. Iglesias, E.L. Souza, R.A. Freitas, H.C. Galvão, Saliva: current methods for collection and attainment of the sample, Rev. Fac. Odontol. Porto Alegre 48 (2007) 95-98

[15] C.P. Schultz, M.K. Ahmed, C. Dawes, H.H. Mantsch, Thiocyanate levels in human saliva: quantitation by Fourier transform infrared spectroscopy, Anal. Biochem. 240 (1996) 7-12

[16] C. Júnior, J.F. Strixino, Raniero L, analysis of saliva by Fourier transform infrared spectroscopy for diagnosis of physiological stress in athletes, Res. Biomed. Eng. 31 (2015) 116-124. 
[17] S. Krimm, J. Bandekar, Vibrational spectroscopy and conformation of peptides, polypeptides, and proteins, Adv. Protein Chem. 38 (1986) 181-364.

[18] A. Michcik, M. Cichorek, A. Daca, P. Chomik, S. Wojcik, A. Zawrocki, A. Wlodarkiewicz, Tobacco smoking alters the number of oral epithelial cells with apoptotic features, Folia Histochem. Cytobiol. 52 (2014) 60-68.

[19] M. Thun, R. Peto, J. Boreham, A.D. Lopez, Stages of the cigarette epidemic on entering its second century, Tob. Control. 21 (2012) 96-101.

[20] D. Hoffmann, I. Hoffmann, K. El-Bayoumy, The less harmful cigarette: a controversia issue. A tribute to Ernst L. Wynder, Chem. Res. Toxicol. 14 (2001) 767-790.

[21] K. Takahashi, S. Yokota, N. Tatsumi, T. Fukami, T. Yokoi, M. Nakajima, Cigarette smoking substantially alters plasma microRNA profiles in healthy subjects, Toxicol. Appl. Pharmacol. 272 (2013) 154-160.

[22] M. Castagnola, P.M. Picciotti, I. Messana, et al., Potential applications of human saliva as diagnostic fluid, Acta Otorhinolaryngol. Ital. 31 (2011) 347-357.

[23] S. Shintani, H. Hamakawa, Y. Ueyama, M. Hatori, T. Toyoshima, Identification of a truncated cystatin SA-I as a saliva biomarker for oral squamous cell carcinom using the SELDI protein Chip platform, Int. J. Oral Maxillofac. Surg. 39 (2010) 68-74.

[24] D.T. Wong, Salivary diagnostics for oral cancer, J Calif Dent Assoc. 34 (2006) 303-308.

[25] N. Robson, A.J. Bond, K. Wolff, Salivary nicotine and cotinine concentrations in unstimulated and stimulated saliva, Afr. J. Pharm. Pharmacol 4 (2010) 61-65.

[26] A. Semlali, J. Chakir, M. Rouabhia, Effects of whole cigarette smoke on human gingival fibroblast adhesion, growth, and migration, J. Toxicol. Environ, Health A 74 (2011) 848-862.

[27] D. Silva, M. Cáceres, R. Arancibia, C. Martínez, J. Martínez, P. Smith, Effects of cigarette smoke and nicotine on cell viability, migration and myofibroblastic differentiation, J. Periodontal Res. 47 (2012) 599-607.

[28] Z.X. Jiao, Q.L. Ao, M. Xiong, Cigarette smoke extract inhibits the proliferation of alveolar epithelial cells and induces apoptosis, Sheng Li Xue Bao 58 (2006) 244-254.

[29] P. Imirzalioĝlu, S. Uckan, E.E. Alaaddinoĝlu, A. Haberal, D. Uckan, Cigarette smoking and apoptosis, J. Periodontol. 76 (2005) 737-739.

[30] C.F. Lima, E. Crastechini, J.S. Issa, I. Balducci, L.A. Cabral, J.D. Almeida, Evaluation of apoptotic pathway in oral mucosa by smoking in a Brazilian outpatient smoking cessation program, Int. J. Cardiol. 184 (2015) 514-516.

[31] N.K. Proia, G.M. Paszkiewicz, M.A. Nasca, G.E. Franke, J.L. Pauly, Smoking and smokeless tobacco-associated human buccal cell mutations and their association with oral cancer: a review, Cancer Epidemiol. Biomark. Prev. 15 (2006) 1061-1077.
[32] J.D. Shulman, M.M. Beach, F. Rivera-Hidalgo, The prevalence of oral mucosal lesions in U.S. adults: data from the Third National Health and Nutrition Examination Survey 1988-1994, J. Am. Dent. Assoc. 135 (2004) 1279-1286.

[33] S. DeFlora, F. D'Agostini, R. Balansky, et al., Modulation of cigarette smoke related end-points in mutagenesis and carcinogenesis, Mutat. Res. 523-524 (2003) 237-252.

[34] A. Besarati Nia, H.W.M. Van Straaten, R.W.L. Godschalk, et al., Immunoperoxidase detection of polycyclic aromatic hydrocarbon-DNA adducts in mouth floor and buccal mucosa cells of smokers and nonsmokers, Environ. Mol. Mutagen. 36 (2000) 127-133.

[35] T.M. Hsu, Y.J. Zhang, R. Stantella, Immunoperoxidase quantitation of 4aminobiphenly- and polycyclic aromatic hydrocarbon-DNA adducts in exfoliated oral and urothelial cells of smokers and nonsmokers, Cancer Epidemiol. Biomark. Prev. 6 (1997) 193-199.

[36] G. Romano, R. Mancini, P. Fedele, et al. Immunohistochemical analysis of 4aminobiphenyl-DNA adducts in oral mucosa cells of smokers and nonsmokers, Anticancer Res. 17 (1997) 2827-2830.

[37] J. Stone, N. Jones, A. McGregor, R. Waters, Development of a human biomonitoring assay using buccal mucosa: comparison of smoking-related DNA adducts in mucosa versus biopsies, Cancer Res. 55 (1995) 1267-1270.

[38] J. Zhou, B.L. Olson, L.J. Windsor, Nicotine increases the collagen-degrading ability of human gingival fibroblasts, J. Periodontal Res. 42 (2007) 228-235.

[39] N.S. Ozek, I. Zeller, et al., Differentiation of chronic and aggressive periodontitis by FTIR spectroscopy, J. Dent. Res. 0022034516663696 (2016).

[40] W. Weuffen, C. Franzke, B. Thürkow, The alimentary ingestion, analysis and biological significance of thiocyanate, Nahrung 28 (1984) 341-355.

[41] F. Pena-Pereira, I. Lavilla, C. Bendicho, Paper-based analytical device for instrumental-free detection of thiocyanate in saliva as a biomarker of tobacco smoke exposure, Talanta 15 (2016) 390-396.

[42] C. Costagliola, L. Cotticelli, M. Menzione, M. Rinaldi, S. Russo, E. Rinaldi, Red cell reduced glutathione and tobacco smoke-induced optic neuropathy, Metab. Pediatr. Syst. Ophthalmol. 13 (1990) 96-98.

[43] L.H. Mosely, F. Finseth, Cigarette smoking: impairment of digital blood flow and wound healing in the hand, Hand 9 (1977) 97-101. 